\title{
Efficacy and safety of chondroitin sulfate/xanthan gum versus polyethylene glycol/propylene glycol/ hydroxypropyl guar in patients with dry eye
}

This article was published in the following Dove Press journal:

Clinical Ophthalmology

28 May 2013

Number of times this article has been viewed

\author{
Juan Francisco Llamas- \\ Moreno' \\ Leopoldo Martín Baiza- \\ Durán' \\ Laura Ray Saucedo- \\ Rodríguez ${ }^{2}$ \\ José Félix Alaníz-De la $\mathrm{O}^{2}$ \\ 'Clinical Research Department, \\ Laboratorios Sophia, SA de \\ CV Zapopan, Jalisco, México; \\ 2Ophthalmology Department, Fray \\ Antonio Alcalde Hospital, Guadalajara, \\ Jalisco, México
}

Purpose: To evaluate the efficacy and safety of two ophthalmic solutions in patients with mild to moderate dry eye.

Methods: We performed a prospective, 2-month-long, randomized, double-blind, single-center, parallel clinical trial to compare the efficacy and safety of two ophthalmic solutions for dry eye treatment. Patients were randomly assigned to one of the two treatment groups, study group or active-control group, and received one drop four times a day. The primary efficacy endpoint was to extend the tear film break-up time (TBUT) after 2 months of treatment. The Ocular Surface Disease Index (OSDI) was also evaluated. Safety measures were assessed by the presence of adverse events.

Results: A total of 28 patients with mild to moderate dry eye were included in the per protocol analysis. TBUT was similar between groups at baseline (chondroitin sulfate and xanthan gum [CS/XG] group, $5.2 \pm 2.3$; Systane ${ }^{\circledR}$ group, $\left.4.7 \pm 2.6 ; P=0.488\right)$, after 2 months of treatment, TBUT was still similar in both groups $\left(\mathrm{CS} / \mathrm{XG}\right.$ group, $6.1 \pm 2.5$; Systane ${ }^{\circledR}$ group, $7.3 \pm 2.5$; $P=0.088$ ). Baseline OSDI was similar between the groups (CS/XG group, $18.8 \pm 5.3$; Systane ${ }^{\mathbb{R}}$ group, $19.8 \pm 7.1 ; P=0.810)$, but after 2 months of treatment, the OSDI was significantly lower in the $\mathrm{CS} / \mathrm{XG}$ group $(6.7 \pm 5.7$ versus $10.8 \pm 6.4 ; P=0.049)$. An adverse event was present in the $\mathrm{CS} / \mathrm{XG}$ group, but it was not related to the treatment.

Conclusions: In this population of patients with mild to moderate dry eye, treatment with $\mathrm{CS} / \mathrm{XG}$ was as effective as treatment with Systane ${ }^{\circledR}$ with regard to TBUT; nevertheless, treatment in the $\mathrm{CS} / \mathrm{XG}$ group was more effective at diminishing OSDI.

Keywords: dry eye, efficacy, safety, TBUT, OSDI

\section{Introduction}

Dry eye is recognized as one of the most common eye diseases, with a high prevalence in many countries. ${ }^{1-3}$ It has been estimated to affect more than 3.2 million middle-aged and older women and nearly 1.7 million men in the United States alone. ${ }^{4}$ Dry eye is a complex disease of inadequate lubrication of the ocular surface and is characterized by ocular irritation resulting from an alteration of the tear film. The effects can vary from a minor inconvenience for most sufferers to rare sight-threatening complications in severe cases. ${ }^{5}$ The International Dry Eye Workshop defined dry eye as a "multifactorial disease of the tears and ocular surface that results in symptoms of discomfort, visual disturbance, and tear film instability, with potential damage to the ocular surface. It is accompanied by increased osmolarity of the tear film and inflammation of the ocular surface." ${ }^{\circ}$ Risk factors for dry eye syndrome include age older than 50 years, female sex, environments with low humidity, systemic medications, autoimmune disorders,
Correspondence: Leopoldo Martín Baiza-Durán

Paseo del Norte No 5255

Guadalajara Technology Park,

Zapopan, CP 45010 Jalisco, México

$\mathrm{Tel}+523330014279$

Fax +523330014200

Email lbaiza@sophia.com.mx 
extensive visual tasking, nutrition (poor intake of fatty acids omega 3, 6 and vitamins A, E), and contact lenses. ${ }^{2,7}$ Patients who suffer from dry eye experience varying levels of dryness, burning, photophobia, foreign body sensation, grittiness, and redness. Patients may or may not have signs that include rapid tear film breakup, increased osmolarity, and increased ocular surface staining. ${ }^{8}$

Various strategies are employed in the treatment and management of dry eye, including the topical use of lubricants (artificial tears or biological tear substitutes) and antiinflammatory therapies, systemic use of antioxidants (eg, omega-3 fatty acids), and control of external factors associated with increased ocular dryness (medications and environmental conditions). ${ }^{8}$ Current treatment is heavily weighted toward supplementation, stimulation, or preservation of aqueous tears..$^{5}$

Artificial tears are one of the primary treatments for dry eye. This treatment is mainly palliative and focuses on reducing the symptoms of discomfort to improve quality of life for a patient. There is no gold standard test to evaluate the efficacy of treatment, nevertheless tear film break-up time (TBUT) is more repeatable than other objective tests for dry eye. ${ }^{9}$

Extending TBUT is the primary aim of artificial tears. Even the best artificial tear provides a limited extension of TBUT (mean extension, usually 5-20 minutes after instillation). ${ }^{10}$

Natural polymers have proved to be effective in sustaining drug releases from a matrix system. ${ }^{11}$ In ocular drugs, the incorporation of polymers in the conventional dosage forms has been found to be an efficient method of increasing the retention time of the dosage form on corneal tissue to enhance the ocular bioavailability. ${ }^{12}$

Xanthan gum (XG) is a high-molecular-weight, watersoluble, heteropolysaccharide of sodium, calcium, or potassium obtained from the aerobic fermentation of a carbohydrate with Xanthomonas campestris. It is a lowviscosity solution that forms viscoelastic gels. The viscous gels reduce the drainage and improve the contact time better than conventional eye drops. XG has been used in combination with other polymers to increase viscosity of the solutions. ${ }^{12,13}$

Chondroitin sulfate (CS) has been used as a lubricant in combination with other components such as sodium hyaluronate for treatment of dry eye ${ }^{14}$ however, there is no previous information about the clinical use of a combination of $\mathrm{CS}$ and $\mathrm{XG}$ in dry eye. This study compared this combination with polyethylene glycol $4000.4 \%$, propylene glycol $0.3 \%$, and hydroxypropyl guar to determine whether $\mathrm{CS} / \mathrm{XG}$ could improve TBUT in patients with dry eye.

\section{Methods}

We conducted a prospective, 2-month-long, parallel, randomized, double-blind, single-center clinical trial to compare the efficacy and safety of two ophthalmic solutions in patients with dry eye disease.

The protocol was reviewed and approved by an ethics committee (CECEIBAC, Guadalajara, Jalisco, México). The research was conducted in compliance with the Declaration of Helsinki and in accordance with Good Clinical Practice Standards. All patients who participated in the study provided written informed consent. The study is registered at ClinicalTrials.gov with the identifier number NCT01541891.

Inclusion criteria for both groups was age 18 years or older, ability to give informed consent, de novo patients diagnosed with mild to moderate dry eye disease based on the Report of International Dry Eye Workshop, ${ }^{6}$ and an Ocular Surface Disease Index (OSDI) score between 12 and 45. ${ }^{15}$ Primary exclusion criteria included patients with one blind eye, visual acuity lower than 20/40 in any eye, any active ocular disease (such as infection or autoimmune inflammation) that would interfere with study interpretation, patients in treatment with any other lubricant or treatment for dry eye disease, contraindication of any medication used in the protocol, history of eye surgery within 3 months before baseline, contact lens use, and patients that were pregnant, at risk for pregnancy without birth control treatment, or breastfeeding.

The primary efficacy endpoint was to evaluate the efficacy (extended TBUT) and safety of two ophthalmic solutions in patients with mild to moderate dry eye.

Patients were assessed for eligibility at a screening visit, when a medical and ocular history was taken. Both eyes were assessed and used for analysis. The screening visit was considered the baseline visit in enrolled patients.

Patients were evaluated during six study visits: at baseline and 2, 7, 15, 30, and 60 days (final visit) after enrollment. Clinical assessments during baseline and the final visit consisted of intraocular pressure (with a calibrated Goldmann applanation tonometer), TBUT, Schirmer I test, indirect ophthalmoscopy under pharmacological mydriasis, OSDI, slit lamp examination (biomicroscopy), and fluorescein and green lissamine dyes. For evaluation of both fluorescein and green lissamine dyes, the Oxford grading scheme was used. ${ }^{6}$ Safety outcome measures included adverse events and ocular signs. 
At each follow-up visit, TBUT, Schirmer I test, biomicroscopy, and fluorescein and green lissamine dyes were performed. Use of concomitant medications was recorded.

Patients were randomly assigned to receive the study medication (study group [CS/XG; PRO-148; Laboratorios Sophia, SA de CV, Guadalajara, Mexico]) or be in the activecontrol group and receive polyethylene glycol $4000.4 \%$, propylene glycol $0.3 \%$, and hydroxypropyl guar (Systane ${ }^{\circledR}$; Alcon Laboratories, Inc, Fort Worth, TX, USA). To maintain masking, medications were bottled and provided in containers that appeared to be identical. To maintain sterility, aseptic techniques were used in a laminar flow cabinet according to good compounding practices. Patients were instructed to instill a single drop of either PRO-148 or Systane ${ }^{\circledR}$ in each eye four times a day for 60 days.

Study treatment was discontinued if either the investigator or the patient thought it was not in the patient's best interest to continue or if the patient became pregnant.

Ocular findings and adverse events regardless of relation to treatment were monitored throughout. The investigators recorded observed adverse events as well as those reported by the patient or elicited by questioning. Adverse events were classified as absent, mild, moderate, or severe, according to the Common Terminology Criteria for Adverse Events v 4.0.

Only randomized patients who concluded the study without a major protocol violation and who provided all measurements at all visits were included in the per protocol analysis.

Statistical analysis was performed using SPSS version 19 (IBM Corporation, Armonk, NY, USA), and differences between treatments in TBUT, OSDI, intraocular pressure, Schirmer I test, and fluorescein and green lissamine dyes were analyzed with a Mann-Whitney $U$ test. A Wilcoxon test was used to evaluate differences between baseline and final evaluations in each group.

Before study initiation, it was determined that at least 15 patients were needed per group, and a significance level of 0.05 and a power of 0.80 were used.

\section{Results}

A total of 30 patients per group were recruited, and a total of 56 eyes of 28 patients were included in the per protocol analysis.

There were no significant differences noted in baseline characteristics between groups (Table 1).

The primary efficacy endpoint was extending TBUT after 2 months of treatment. TBUT was similar between groups at baseline (PRO-148, 5.2 \pm 2.3 ; Systane $^{\circledR}, 4.7 \pm 2.6 ; P=0.488$ ),
Table I Summary of patient baseline characteristics between study groups

\begin{tabular}{lrrl}
\hline Characteristic & PRO-I 48 & Systane $^{\circledR}$ & $\boldsymbol{P}$ \\
\hline Age & $55.8 \pm 8.4$ & $55.9 \pm 9.6$ & 0.710 \\
TBUT & $5.2 \pm 2.3$ & $4.7 \pm 2.6$ & 0.488 \\
Schirmer I test & $8.5 \pm 4.7$ & $9.7 \pm 3.4$ & 0.092 \\
Total corneal staining score & $1.2 \pm 1.8$ & $1.8 \pm 2.8$ & 0.943 \\
Total conjunctival staining score & $0.5 \pm 1.0$ & $0.9 \pm 1.6$ & 0.407 \\
Global staining score & $1.7 \pm 1.7$ & $2.7 \pm 4.0$ & 0.970 \\
OSDI & $18.8 \pm 5.3$ & $19.8 \pm 7.1$ & 0.810
\end{tabular}

Note: Data are presented in mean \pm standard deviation.

Abbreviations: TBUT, tear film break-up time; OSDI, Ocular Surface Disease Index.

and after 2 months of treatment, TBUT was similar in both groups (PRO-148, $6.1 \pm 2.5$; Systane ${ }^{\circledR}, 7.3 \pm 2.5 ; P=0.088$ ). Although there was a mild increase in TBUT in both groups when compared with baseline, this increase was not statistically significant (PRO-148, $5.2 \pm 2.3$ versus $6.1 \pm 2.5[P=0.222]$; Systane $^{\circledR}, 4.7 \pm 2.6$ versus $7.3 \pm 2.5[P=0.321]$; Figure 1$)$.

Baseline OSDI was similar between groups (PRO-148, $18.8 \pm 5.3$; Systane $^{\circledR}, 19.8 \pm 7.1 ; P=0.810$ ). At study conclusion, OSDI was reduced in both groups when compared with baseline, and this reduction was statistically significantly lower in the PRO-148 group compared with the Systane ${ }^{\circledR}$ group $(6.7 \pm 5.7$ versus $10.8 \pm 6.4 ; P=0.049$; Figure 2). The rest of the study measurements did not present differences between the groups.

With regard to Schirmer I and staining scores, there were no differences between groups.

There was a single adverse event present in a patient from the PRO-148 group; this event, a mild-intensity cephalea at visit 1 that did not need medications for its relief, was not related to the treatment. The rest of the patients in both groups did not present any adverse events.

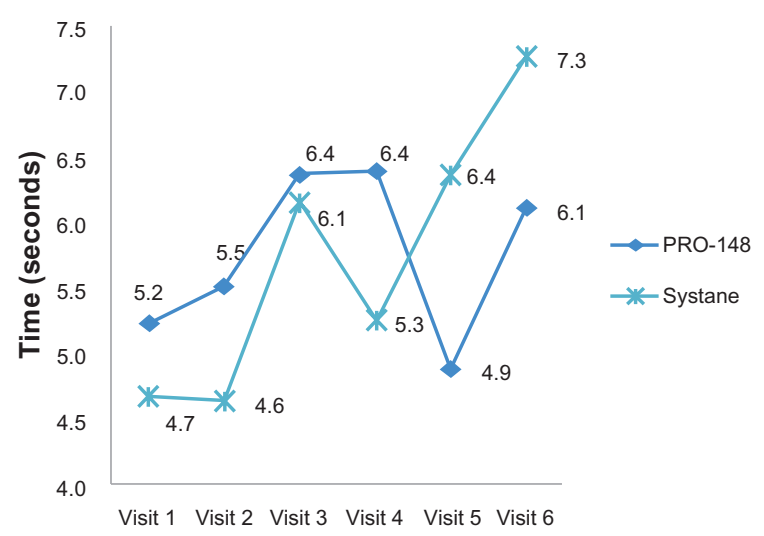

Figure I Mean change from baseline tear film break up time at each visit between groups. 


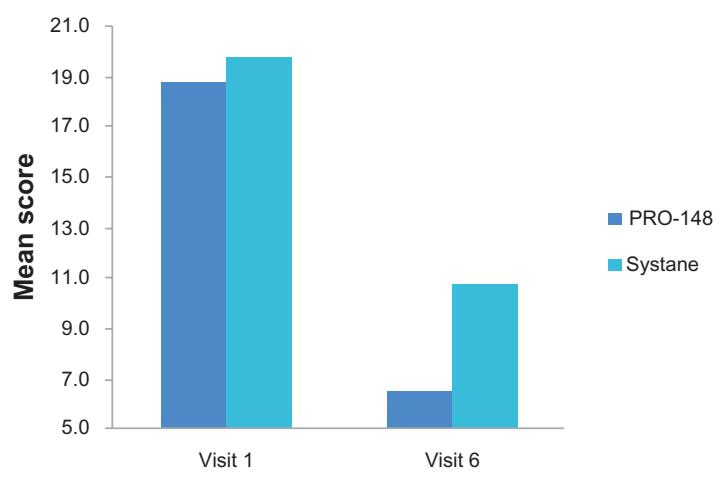

Figure 2 Mean change from baseline Ocular Surface Disease Index at each visit between groups.

\section{Discussion}

Dry eye results in disruption of the tear film and subsequent damage to epithelial cells on the ocular surface, which may exacerbate signs and symptoms of the disease experienced by the patient. Significant advances have been made in treating the many facets of dry eye; current therapy focuses on restoring a normal ocular surface through tear supplementation as well as inhibition of the aberrant inflammation seen in chronic dry eye disease. Regardless of the etiology and severity of dry eye, artificial tears are the first line of treatment. ${ }^{5,16}$ Commercial artificial tears differ in electrolyte composition, thickening agents, physiologic buffering, tonicity, and preservatives. ${ }^{5}$

Studies in which treatments for dry eye have been compared show us that some have better efficacy than others. Although TBUT is the best screening test for dry eye disease and is more repeatable - compared with other objective tests for dry eye, ${ }^{9}$ the OSDI has shown good reliability and validity for measuring the severity of dry eye disease and has been proposed as a valuable tool in clinical treatment trials. ${ }^{1,6}$

There is no previous information about the clinical use of $\mathrm{CS}$ and XG in dry eye. Nevertheless, there are some studies in which other combinations have been compared. For example, Davitt et $\mathrm{al}^{8}$ compared carboxymethylcellulose $0.5 \%$, glycerin $0.9 \%$, and compatible solutes with polyethylene glycol $4000.4 \%$, propylene glycol $0.3 \%$, and hydroxypropyl guar. In this study, both groups reported a significant reduction in the mean scores for the ocular symptoms of dryness, gritty/ sandy feeling, and burning. Ocular staining scores were lower in the polyethylene glycol $4000.4 \%$, propylene glycol $0.3 \%$, and hydroxypropyl guar group at days 28 and 42 . For TBUT there was no difference between treatments, but mean OSDI was significantly reduced in both groups.

A review by Benelli ${ }^{17}$ concluded that a solution of polyethylene glycol $4000.4 \%$, propylene glycol $0.3 \%$, and hydroxypropyl guar offers extended relief of dry eye symptoms and improvement of the signs associated with dry eye disease. In this review, this combination exhibits a superior profile in TBUT, extensional viscosity, coefficient of friction and lubrication, and conjunctival and/or corneal staining when compared with other marketed products.

Another study by Gensheimer et $\mathrm{al}^{18}$ evaluated the effectiveness of glycerin $1 \%$ with a new excipient in extending TBUT compared with polyethylene glycol 400 $0.4 \%$, propylene glycol $0.3 \%$ and hydroxypropyl guar. In this study the formulation of glycerin $1 \%$ with the new excipient significantly extended TBUT.

Faraldi et al ${ }^{19}$ investigated an ophthalmic gel containing sodium hyaluronate and $\mathrm{XG}$ for the management of posttraumatic corneal abrasions. In this study, the author demonstrated that sodium hyaluronate and $\mathrm{XG}$ are highmolecular-weight polysaccharides with water binding and mucoadhesivity properties, providing effective hydration and protection of the cornea.

The present study compared two ophthalmic solutions, one containing $\mathrm{CS}$ and $\mathrm{XG}$ and the other containing polyethylene glycol $4000.4 \%$, propylene glycol $0.3 \%$, and hydroxypropyl guar. We demonstrated that the new combination (PRO-148) effectively reduced OSDI and is similar in regard to TBUT when compared with Systane ${ }^{\circledR}$.

In this reduced population of patients with dry eye, both groups demonstrated a reduction of OSDI scores toward the normal range, with a slight difference favoring the test group.

The limitations of our study are that we only included a small number of patients with mild to moderate dry eye, and we did not conduct a crossover or internal control study. Larger studies with longer duration and employing different designs are warranted to assess the long-term safety and efficacy of this formulation in patients with dry eye disease.

\section{Conclusion}

Increasing TBUT is important in the treatment of dry eye disease because it can improve clinical signs and symptoms of dry eye disease and promote epithelial repair. The goal of topical therapy in dry eye is to relieve symptoms in conjunction with potentially replacing deficient components (eg, aqueous fluid) so that the tear film is thickened and stabilized in the short-term to provide healing of the ocular surface over time. The OSDI permits quantification of common symptoms and an objective approach to the evaluation of symptoms over time; therefore, OSDI reduction could indicate that treatment goals are achieved. 
In the present study, the combination CS and XG was as effective in prolonging TBUT and was better at diminishing OSDI compared with polyethylene glycol $4000.4 \%$, propylene glycol $0.3 \%$, and hydroxypropyl guar.

\section{Disclosure}

This study was sponsored by Laboratorios Sophia, SA de CV. The authors are employees of Laboratorios Sophia, SA de CV. The authors report no other conflicts of interest in this work.

\section{References}

1. Bhavsar AS, Bhavsar SG, Jain SM. A review on recent advances in dry eye: Pathogenesis and management. Oman J Ophthalmol. 2011;4(2): 50-56.

2. Brujic M. 11th annual dry eye report recalibrate dry eye management [webpage on the Internet]. Newtown Square, PA: Review of Optometry; 2010. Available from: http://www.revoptom.com/continuing_education/ tabviewtest/lessonid/106485/. Accessed January 24, 2013.

3. Mizuno Y, Yamada M, Shigeyasu C. Annual direct cost of dry eye in Japan. Clin Ophthalmol. 2012;6:755-760.

4. Schaumberg DA, Dana R, Buring JE, Sullivan DA. Prevalence of dry eye disease among US men: estimates from the Physicians' Health Studies. Arch Ophthalmol. 2009;127(6):763-768.

5. Yanoff M, Duker JS, editors. Ophthalmology, 3rd ed. Edinburgh, Scotland: Mosby; 2009.

6. The definition and classification of dry eye disease: report of the Definition and Classification Subcommittee of the International Dry Eye WorkShop (2007). Ocul Surf. 2007;5(2):75-92.

7. Yao W, Davidson RS, Durairaj VD, Gelston CD. Dry eye syndrome: an update in office management. Am J Med. 2011;124(11):1016-1018.
8. Davitt WF, Bloomenstein M, Christensen M, Martin AE. Efficacy in patients with dry eye after treatment with a new lubricant eye drop formulation. J Ocul Pharmacol Ther. 2010;26(4):347-353.

9. Nichols KK, Mitchell GL, Zadnik K. The repeatability of clinical measurements of dry eye. Cornea. 2004;23(3):272-285.

10. Ousler GW, Michaelson C, Christensen MT. An evaluation of tear film breakup time extension and ocular protection index scores among three marketed lubricant eye drops. Cornea. 2007;26(8):949-952.

11. Rajesh KS, Venkataraju MP, Gowda DV. Effect of hydrophilic natural gums in formulation of oral-controlled release matrix tablets of propranolol hydrochloride. Pak J Pharm Sci. 2009;22(2):211-219.

12. Pahuja P, Arora S, Pawar P. Ocular drug delivery system: a reference to natural polymers. Expert Opin Drug Deliv. 2012;9(7):837-861.

13. Li H, Chen R, Lu X, Hou W. Rheological properties of aqueous solution containing xanthan gum and cationic cellulose JR400. Carbohydr Polym. 2012;90(3):1330-1336.

14. Moon JW, Lee HJ, Shin KC, Wee WR, Lee JH, Kim MK. Short term effects of topical cyclosporine and viscoelastic on the ocular surfaces in patients with dry eye. Korean J Ophthalmol. 2007;21(4): 189-194.

15. Ozcura F, Aydin S, Helvaci MR. Ocular surface disease index for the diagnosis of dry eye syndrome. Ocul Immunol Inflamm. 2007;15(5): 389-393.

16. Springs CL. Novel hydroxypropyl-guar gellable lubricant eye drops for treatment of dry eye. Adv Ther. 2010;27(10):681-690.

17. Benelli U. Systane lubricant eye drops in the management of ocular dryness. Clin Ophthalmol. 2011;5:783-790.

18. Gensheimer WG, Kleinman DM, Gonzalez MO, et al. Novel formulation of glycerin $1 \%$ artificial tears extends tear film break-up time compared with Systane lubricant eye drops. J Ocul Pharmacol Ther. 2012;28(5): 473-478.

19. Faraldi F, Papa V, Santoro D, et al. A new eye gel containing sodium hyaluronate and xanthan gum for the management of post-traumatic corneal abrasions. Clin Ophthalmol. 2012;6:727-731.
Clinical Ophthalmology

\section{Publish your work in this journal}

Clinical Ophthalmology is an international, peer-reviewed journal covering all subspecialties within ophthalmology. Key topics include: Optometry; Visual science; Pharmacology and drug therapy in eye diseases; Basic Sciences; Primary and Secondary eye care; Patient Safety and Quality of Care Improvements. This journal is indexed on

Submit your manuscript here: http://www.dovepress.com/clinical-ophthalmology-journal

\section{Dovepress}

PubMed Central and CAS, and is the official journal of The Society of Clinical Ophthalmology (SCO). The manuscript management system is completely online and includes a very quick and fair peer-review system, which is all easy to use. Visit http://www.dovepress.com/ testimonials.php to read real quotes from published authors. 